\title{
SOC IMPLEMENTATION OF CAN CONTROLLER
}

\author{
Sayali Chalke, Kirti Bhosale, Gargi Rege, Prof. Ashish Vartak \\ Electronics Engineering \\ Finolex Academy of Management and Technology, Ratnagiri
}

\begin{abstract}
Now day's communications plays a crucial role in the era of internet. On internet most of the data is in the form of images and videos. For better quality of service (QoS) speed is main factor which actively takes part in uploading and downloading. To achieve such aspect Controller Area Network (CAN) plays a vital role.

CAN controller receive and transmits a data upto $1 \mathrm{Mbps}$ speed. It is most reliable proposal for automobile applications like VANET. Due to fruitful features of CAN, it becomes very popular in Industrial-Scientific-Medical (ISM) platform. The CAN controller decreases the wiring complexity and make it easy to connect other devices using single pair of wires, allowing the data exchange between them at the same time. Its cost performance and upgradability provides various flexibility in system design. This review presents brief outlook of CAN protocol attainments in various hardware like processors and all other connected peripherals and software. In this paper we take a brief review regarding CAN controller and CAN communication protocol.
\end{abstract}

Keywords - Controller Area Network protocol, FPGA, ARM.

\section{INTRODUCTION}

The Controller Area Network is a method of communication between various electronics devices like engine management systems, gear control, lighting control, air conditioning, air bag, etc. embedded in an automobile. The idea of controlled area network (CAN) was developed by Robert Bosch GmbH of Germany in the early 1980s. The CAN bus presents different modules of interconnection where each module communicates with each other under the ideal platform. It is also used in higher data integrity networked system for fast and robust communication. Installation and utilization of CAN network is simple and inexpensive circuitry. It specifically operates within noisy environment of control system for electronics units and parameters. CAN acts as transmission protocol which is message oriented. After every transmission the frame content is checked by a Cyclic Redundancy Code (CRC) mechanism. The incorrect message will be ignored by the nodes. In the case of too many errors the nodes can disconnect themselves or even stop transmitting messages.

CAN has various properties such as prioritization of messages, guarantee of latency times, flexibility, multicast reception with time synchronization, multimaster error detection and signaling, automatic retransmission of corrupted messages as soon as the bus is idle again. Such characteristics are very attractive to make CAN a suitable communication protocol for sensors networks wired and wireless. This protocol efficiently support distributed real time control with a very high level of security. Currently CAN is also used in other applications, such as: home systems, medical devices, industrial control, etc. The interest in CAN is increasing rapidly due to the different applications that are forces and availability of devices integrating CAN in the market .CAN protocol use in electronic equipment for aviation and navigation it is use in industrial automation and mechanical control. The modern automobile may have various electronic control units for various subsystems. Processor is the engine control unit. Others are used for transmission, airbags, antilock braking /ABS, cruise control, electric power steering, electric cars, etc.

\section{BLOCK DIAGRAM OF CAN CONTROLLER}

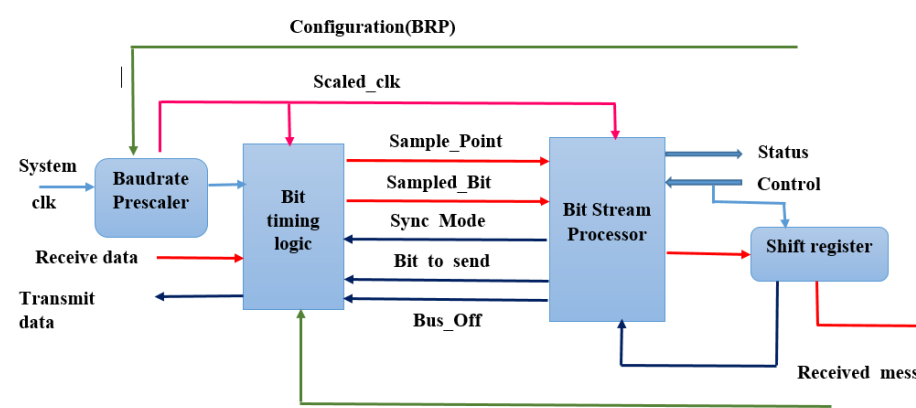

Fig.1 CAN Controller

Bit timing logic:-

This unit configure the timing parameter of the bit time for the CAN node. Each CAN node is configured individually to create a common bit rate for all the nodes on the network even though the CAN nodes oscillator period may be different. Synchronization and resynchronizations are perform on the receive dominant transition edges. The purpose is to control the distance between edges and sample points.

\section{Boudrate Prescaler:-}

The Baudrate Prescaler for the oscillator or internal clock sets the time quantum and the bit time is multiple of (TQ).The hardware selection of the oscillator and the software configuration of the BRP and number of TQ per bit time set the data rate. 


\section{Bit Stream Processor:-}

BSP is a sequencer controlling the sequential data stream between transmitter and receiver shift register the CRC register and the bus line. The BSP also controls the Error Management Logic and the parallel data stream between the transmitter and receiver shift register and the intelligent memory such that the processes of reception, arbitration, transmission and error signaling are performed according to the CAN controller.

\section{Shift Register:-}

Shift register commonly used in converters that translates parallel data, or vice versa. It can also function as delay circuits and digital pulse extender. It is mostly used for data storage or for the movement of data and are therefore commonly used inside calculators or computers to store data such as two binary numbers before they are added together.

\section{BACKGROUND}

Rugved D. Katyarmal e al., Controller area network is designed for sensor network applications like weather monitoring, Automation industries etc. For such applications CAN is designed by using Verilog-HDL. Communication modules are required for sensors interface with the sensor network. CAN provide a high decreasing in wiring complexity and additionally make it easy to connect with several devices using a single pair of wires allowing the data exchange between them at the same time. The CAN controller has been designed in Verilog. The design is implemented on FPGA. The designed CAN Controller is interface with a LM35 temperature sensor.[1]

Amrutha E.et al., CAN protocol is designed for greenhouse system. In this paper and automatic greenhouse monitoring and control system based on CAN protocol and mobile web server has been design. This system increase for lot of agriculture products has created awareness for the farmers to increase their production by implementing the latest technology in the field. The objective is to develop a system that concentrates on providing the means for the management of the environment and crop growth information. The proposed system would be economical effective and easy to install. The microclimatic parameters that are generally consider for monitoring and control are temperature, humidity, light intensity, moisture content in the soil, carbon dioxide (CO2) etc.[2]

Sheher Banu et al., The designed of accident avoidance system in automobiles using CAN protocol. Basic requirement and advancement in the technology in the field of vehicles safety has led to the implementation of the CAN protocol to avoid the accident. Advantage of CAN based design provides flexibility and expandability for technology insertions. Sensors are used to measure the various parameters in a vehicles like speed, distance between vehicles existing of alcoholic person in the vehicle and sending a warning signal to the driver if any one of the parameters varies, along with that detecting the collisions and mechanical shocks to the vehicles sense by the sensor during the accident and sending message to the any predefine numbers. Apart from this on occurrence of any accident in any remote area then vibration sensor detects the vehicles collision and sends SMS immediately via GSM along with exact location provided by GPS to make the system completely reliable, safe and stable. [3]

Ashwini S. Shinde et al., it is different automobile application in CAN Protocol. The design and implementation of CAN bus prototype in ARM 7 processor for vehicle automation. This automation techniques spotlights on both hardware and software design nodes with all intelligence. Interfaces in hardware circuit mainly consist of MCP2515, LPC2148 microcontroller. MPC2515 and MCP21 are standalone CAN controller with SPI interface and high speed CAN transceiver respectively. This proposed software design is implemented for CAN bus network to design CAN bus data communication and data processing for analog signal between nodes. This design consist of software design communication module which comprises system initialization unit, CAN controller initialization unit, message sending and receiving unit and the interrupt service unit.[4]

Ashish P. Patil et al., CAN protocol designed for vehicle brake control system at slope. Generally there are two types of brake systems in vehicles. One is disc brake and other one is box brake. Both the types of brakes in the vehicle are control using the CAN protocol. When the vehicles are moving in slope, its brake system is control manually. This system introduces a technique to control those vehicles moving in slope automatically with the help of CAN protocol.PIC based data acquisition system is used that uses ADC to bring all control data from analog to digital format. Since the information systems in a vehicle are spread throughout the practical vehicle. The communication module used here is embedded networking by CAN.[5]

Tejal Farkande et al., developed CAN to employ a cost effective communication bus in cars. This paper also present successful extraction o CAN messages from automobile ECU's. The extraction is perform by building hardware and software, also projects interfacing and communication directly with embedded CAN network. Implementation of this process give rise to extracted CAN messages which is used for communication in automobile ECU's. The above data been user to keep updating status of automobile system. [6] 


\section{International Journal of Engineering Applied Sciences and Technology, 2018 \\ Vol. 3, Issue 10, ISSN No. 2455-2143, Pages 23-25 \\ Published Online February 2019 in IJEAST (http://www.ijeast.com)}

Vinodh Kumar et al., implemented strategies of automotive deals with short messaging schemes and selective bit stuffing method to improve the effective utilization of network bandwidth. This paper present a work on short CAN technique for effective utilization of available CAN bandwidth. It also discovers the impact of selective bit stuffing over data payload without any major change in the exciting CAN protocol all the above performance of working model simulation and real time CANoe hardware with multi functional display proving bandwidth utilization. [7]

Manuele Bertoluzzo et al., analysed the limitations of CAN and has provided two protocol has solutions: time triggered protocols and native CAN with ad-hoc developed application protocol. The protocol, termed time triggered bus-redundant CAN is built up around the time triggered concept and bus redundancy. Thus this paper provides novel of CAN application protocol with performance of the existing ones in time determinism and processing time. [8]

Santosh kumar et al., propose work, where is measure are converted into two modules as master module and slave module. The propose automatic front headlight adjustment system is done by master module consist of LPG gas leakage sensor and temperature monitoring unit. Other sensors like digital fuel level sensor, radio frequency transmitter and receiver are used for horn volume automatic adjustment in slave module. The controlled area network communication between master and slave are achieve with high reliability this paper also projected cost effective and improved network speed of $1 \mathrm{Mb} / \mathrm{s}$, with excellent error detection and reduce hardware size. [9]

Samuel Woo et al., derives an attack model using a malicious smart-phone application. This proposed attack model processed in the connected car environment are demonstrated practically o research. Thus security protocol in the paper designed and applied to the car environment with an analysis of the vulnerability of in-vehicle CAN. [10]

\section{CONCLUSION}

The review of a CAN controller has been reported in this paper. CAN protocol initially developed for in-vehicle networking of automobile applications in various other industries. CAN is ideally suited in applications requiring a large number of short messages with high reliability in rugged operating environments.

\section{REFERENCES}

[1] Chen H., and Tian J.(2009) "Re+search on the controller Area Network",(pp.251-254).
[2] Ranjan R., and Chari K. S. (2015) “ Design of controller Area Network Based Automated Safety System for networking systems for lighting control application" ,(pp.1301-1306).

[3]Scholar P. "Autonomous All-Terrain Vehicle using CAN bus",(p.5).

[4]Vidyasagar B.(2012) "Greenhouse Monitoring and Automation using GSM", International Journal of scientific and Research Publications, (vol2)

[5]Shinde A.S.(2012) "Controller Area Network for Vehicle Automation”,(vol. 2, no. 2, p. 6)

[6]Pathyil E.(2009) "ECSE Design Final Report:Controller Area Network(CAN)Version1.6.,"Rensselaer Polytechnic Institute, Troy

[7]Burje P., and Karande K.(2014) "Embedded On-Board Diagnostics System using CAN Protocol,’(pp. 734-737).

[8]Varghese S., and Kurian C.(2015) "A study of communication protocols and wireless networking system for lighting control application,"(pp. 1301-1306).

[9]Devi A., and Gnanavel G.(2014) "MCS 51 Microcontroller based Industrial Automation and Control System using CAN protocol, "(pp. 061-065).

[10]Farkande T., and Pawar S. "Controller Area Network Data Extraction for Automobile," (vol.3 no.1 p.6). 